MATHEMATICS OF COMPUTATION

Volume 80, Number 276, October 2011, Pages 2281-2287

S $0025-5718(2011) 02472-5$

Article electronically published on March 9, 2011

\title{
AN IMPROVED LOWER BOUND FOR THE DE BRUIJN-NEWMAN CONSTANT
}

\author{
YANNICK SAOUTER, XAVIER GOURDON, AND PATRICK DEMICHEL
}

\begin{abstract}
In this article, we report on computations that led to the discovery of a new Lehmer pair of zeros for the Riemann $\zeta$ function. Given this new close pair of zeros, we improve the known lower bound for de Bruijn-Newman constant $\Lambda$. The Riemann hypothesis is equivalent to the assertion $\Lambda \leq 0$. In this article, we establish that in fact we have $\Lambda>-1.14541 \times 10^{-11}$. This new bound confirms the belief that if the Riemann hypothesis is true, it is barely true.
\end{abstract}

\section{INTRODUCTION}

The Riemann hypothesis is a long standing conjecture in mathematics. It asserts that the non-trivial zeros of the Riemann $\zeta$ function all have a real part equal to $1 / 2$. Many attempts have been made to prove it. To this end, Pólya considered a family of trigonometric integrals $H_{\lambda}$, defined by

where

$$
H_{\lambda}(x)=\int_{0}^{\infty} e^{t u^{2}} \Phi(u) \cos (x u) d u \quad(\lambda \in R, x \in C),
$$

$$
\Phi(u)=\sum_{n=1}^{\infty}\left(2 \pi^{2} n^{4} e^{9 u}-3 \pi n^{2} e^{5 u}\right) \exp \left(-\pi n^{2} e^{4 u}\right) \quad(0 \leq u<\infty) .
$$

With this definition, we have [Tit86, p. 255] $H_{0}(x)=\Xi(x / 2) / 8$, where $\Xi$ is the classic Riemann function defined by

$$
\begin{aligned}
& \Xi(x)=-\frac{1}{2}\left(x^{2}+\frac{1}{4}\right) \xi\left(\frac{1}{2}+i x\right), \\
& \xi(s)=\frac{1}{2} s(s-1) \pi^{-s / 2} \Gamma\left(\frac{1}{2} s\right) \zeta(s) .
\end{aligned}
$$

The Riemann hypothesis is then equivalent to the fact that the functions $\Xi$ and $H_{0}$ have only real zeros. Pólya hoped to prove this property for the integral functions $H_{\lambda}$, and to deduce the Riemann hypothesis as a corollary. In the general case, results were obtained by de Bruijn and Newman. In fact, de Bruijn dB50 showed that $H_{\lambda}$ has only real zeros if $\lambda \geq 1 / 2$. Moreover, if $\lambda$ is such that $H_{\lambda}$ has only real zeros, then it also holds for any $\lambda^{\prime}$ with $\lambda^{\prime} \geq \lambda$. On the other hand, Newman [New76] showed that there is at least one value for $\lambda$ such that the corresponding $H_{\lambda}$ function has at least one non-real zero. Thus the following definition makes sense.

Received by the editor May 7, 2009 and, in revised form, August 6, 2010.

2010 Mathematics Subject Classification. Primary 11-04, 11M26, 11Y35, 11 Y60.

(C)2011 American Mathematical Society 
Definition 1.1. There exists a finite real value $\Lambda$ such that $H_{\lambda}$ has only real zeros if and only if $\lambda \geq \Lambda$. The value $\Lambda$ is called the de Bruijn-Newman constant.

With this definition, the Riemann hypothesis is equivalent to the assertion $\Lambda \leq 0$. Recently, Kai et al. KKL09 have shown that $\Lambda<1 / 2$. Much work has been done to obtain lower bounds for $\Lambda$ CNV88, tR91, NRV92, CRV91]. These were based on the search of non-real zeros for Jensen polynomials, except for CRV91, which involves the computation of the Laguerre difference for functions $H_{\lambda}$. Then, Csordas et al. CSV94 made studies on the law of motion for the zeros of functions $H_{\lambda}$ when $\lambda$ is varying. They obtained a new lower bound for $\Lambda$ by considering a close pair of zeros for the function $\zeta$. Such pairs, whose difference is unusually small, are called Lehmer pairs. Then successive computations of zeros for $\zeta$ gave improved lower bounds for $\Lambda$ CSV94, COSV93, Odl00. In Odl00, Odlyzko obtained the best lower bound, prior to the work related here, by establishing that $\Lambda>-2.7 \times 10^{-9}$.

\section{The de Bruijn-Newman constant and Lehmer pairs}

The basic tool that we will use in this article is the main theorem of CSV94 (Theorem $\mathrm{A}$ below). As we will see, this theorem enables us to obtain only negative lower bounds for $\Lambda$. Thus, without explicit mention to the contrary, in this paper, we always suppose that the Riemann hypothesis holds. If it is not the case, then we have $\Lambda>0$ and thus any negative lower bound we might obtain for $\Lambda$ is trivially correct. Likewise, an eventual multiple zero for $H_{0}$ would immediately prove that $\Lambda \geq 0$ [CSV94. Thus, we also suppose that the zeros of $H_{0}$ are all real and simple. Note that if $x$ is a zero, then $-x$ is also a zero. Therefore, we number the positive zeros of $H_{0}$ in increasing order:

$$
0<x_{1} \leq x_{2} \leq x_{3} \leq x_{4} \leq \ldots
$$

and we extend our numbering to negative zeros by putting

$$
x_{-j}=-x_{j} \quad(j=1,2, \ldots),
$$

with $x_{0}$ undefined. With the definition of $H_{0}$, we note that the zeros of the Riemann $\zeta$ function are exactly $1 / 2+i x_{j} / 2$ for $j \in \mathbb{Z}-0$. Given this, following CSV94, we define Lehmer pairs of zeros.

Definition A. Let $k$ be a positive integer such that $x_{k}$ and $x_{k+1}$ are successive simple zeros of $H_{0}$. Then $\left(x_{k}, x_{k+1}\right)$ is a Lehmer pair of zeros for $H_{0}$ if we have $\left(x_{k+1}-x_{k}\right)^{2} \cdot G_{k}<4 / 5$ with

$$
G_{k}=\sum_{j \neq 0, k, k+1}\left\{\frac{1}{\left(x_{k}-x_{j}\right)^{2}}+\frac{1}{\left(x_{k+1}-x_{j}\right)^{2}}\right\} .
$$

We can now state the main theorem from [CSV94.

Theorem A. Let $\left(x_{k}, x_{k+1}\right)$ be a Lehmer pair of zeros for $H_{0}$. Set

$$
\lambda_{k}=\frac{\left(1-\frac{5}{4}\left(x_{k+1}-x_{k}\right)^{2} \cdot G_{k}\right)^{4 / 5}-1}{8 G_{k}}
$$

so that $-1 /\left(8 G_{k}\right)<\lambda_{k}<0$. Then the de Bruijn-Newman constant $\Lambda$ satisfies the inequality $\Lambda \geq \lambda_{k}$.

The Taylor expansion for $\lambda_{k}$ shows that, provided that $G_{k}$ is not too large, $\lambda_{k}$ is close to $-\left(x_{k+1}-x_{k}\right)^{2} / 8$. In this case, the closer a Lehmer pair, the better the lower bound. 
TABLE 1. Close pairs of zeros for the function $\zeta(1 / 2+i t)$, according to Gourdon and Demichel GD04.

\begin{tabular}{|r|r|r|}
\hline$t_{1}$ & $t_{2}$ & $t_{2}-t_{1}$ \\
\hline 7954022502373.43289015387 & 7954022502373.43289494012 & $4.7863 \times 10^{-6}$ \\
\hline 18580341990011.1593414105 & 18580341990011.1593364110 & $4.9995 \times 10^{-6}$ \\
\hline 18523741991636.3643689901 & 18523741991636.3643759580 & $6.9679 \times 10^{-6}$ \\
\hline 4307762397492.0712366929 & 4307762397492.0712295734 & $7.1195 \times 10^{-6}$ \\
\hline 16671318581127.6545668867 & 16671318581127.6545740724 & $7.1857 \times 10^{-6}$ \\
\hline 8847150598019.2235982778 & 8847150598019.2236059816 & $7.7038 \times 10^{-6}$ \\
\hline 10123949469608.2309097616 & 10123949469608.2309189455 & $9.1839 \times 10^{-6}$ \\
\hline 20825125156965.3882387859 & 20825125156965.3882484837 & $9.6978 \times 10^{-6}$ \\
\hline 17335277232221.2458033031 & 17335277232221.2457934935 & $9.8096 \times 10^{-6}$ \\
\hline 5907264585921.6903635665 & 5907264585921.6903535057 & $1.00608 \times 10^{-5}$ \\
\hline 21285800773585.4550406956 & 21285800773585.4550510671 & $1.03715 \times 10^{-5}$ \\
\hline
\end{tabular}

\section{Numerical Verification of the Riemann hypothesis}

Much work has been done on the numerical verification of the Riemann hypothesis. For what we are concerned with here, those computations are useful to obtain Lehmer pairs of zeros. However, from a more general point of view, those computations can be used, for instance, to improve bounds on Chebyshev functions or to broaden zero free regions for $\zeta$. The largest systematic verification with an official announcement was performed in 2001 by van de Lune vdL01. He claimed that the first $10^{10}$ zeros of the function $\zeta$ lie on the critical line and they are all simple. The verification performed was systematic up to height $T=3293531632.415$. These numerical values will be used in what follows.

Two other larger verifications have been performed but neither obtained an official certification. The first one was managed by Wedeniwski [Wed05], and involved distributed computations over more than ten thousands computers. After four years of computation, the project was ended and Wedeniwski announced that the first $10^{11}$ zeros of the Riemann $\zeta$ function lie on the critical line. Computations were based on the Riemann-Siegel formula. The second verification was by Gourdon and Demichel GD04. Using the fast multiple evaluation algorithm of Odlyzko OS88, in 2004, they verified that the first $10^{13}$ zeros lie on the critical line. Moreover, Demichel continued computing and reached the $10^{14}$-th zero. They obtained many pairs of close zeros (see Table 11).

However, neither of these verifications was validated by the American Mathematical Society. The reason seems to be, in Wedeniwski's case, that there was no evidence that all zeros were computed. Also, the verification of Gourdon and Demichel had not been checked independently. In this paper, we refer to close zeros obtained by Gourdon and Demichel. Thus, in order to formally establish our new lower bound, we will have to verify their calculations, at least near the close pair of zeros we will consider.

\section{An UPPER BOUND FOR $G_{K}$}

In order to establish the new lower bound, we will use Theorem $\mathrm{A}$ with the first close pair of Table 1. In the following, we denote the rank of appearance of this 
TABLE 2. A close pair of zeros for $\zeta$, with adjacent zeros.

\begin{tabular}{|r|r|}
\hline Gram blocks & Zeros \\
\hline$\left[g_{34011300784333}, g_{34011300784335}\right)$ & $t_{K-2}=7954022502373.0094352194$ \\
& $t_{K-1}=7954022502373.1219461606$ \\
\hline$\left[g_{34011300784335}, g_{34011300784337}\right)$ & $t_{K}=7954022502373.43289015387$ \\
& $t_{K+1}=7954022502373.43289494012$ \\
\hline$\left[g_{34011300784337}, g_{34011300784338}\right)$ & $t_{K+2}=7954022502373.7336782379$ \\
\hline
\end{tabular}

pair as $K$. This means that $x_{K}=2 t_{1}$ and $x_{K+1}=2 t_{2}$ where the values $t_{1}$ and $t_{2}$ refer to the champion pair of Table 1. We will then have to verify that this pair of zeros is indeed a Lehmer pair. The purpose of this part is to obtain an upper bound for $G_{K}$ that only depends on $x_{K-1}, x_{K}, x_{K+1}$ and $x_{K+2}$.

The main theorem that we will use to validate our new lower bound is the following (see COSV93]).

Theorem B. If $K>10^{10}$, then

$$
\begin{aligned}
G_{K} \leq & \frac{2 \log \left(x_{K+1}\right)}{\left(x_{K+1}-x_{K+2}\right)^{2}}+\frac{2 \log \left(x_{K}\right)}{\left(x_{K}-x_{K-1}\right)^{2}} \\
& \quad+\left(\log \left(x_{K}\right)+\log \left(x_{K+1}\right)\right) \frac{\pi^{2}}{12}+\frac{4 \times 10^{10}}{\left(x_{K}-2 T\right)^{2}}+4 .
\end{aligned}
$$

Proof. Analytical computations related to the proof of this theorem can be found in COSV93. The major difference here, concerns $K$, which is treated as an unknown in Theorem B while it has a fixed value in COSV93.

\section{A New Bound for the De Bruijn-Newman constant}

With Theorem B, for a given close pair of zeros $\left(t_{K}, t_{K+1}\right)$ of $\zeta$, it is possible to compute an upper bound for $G_{K}$ if we also have numerical values for the two adjacent zeros $t_{K-1}$ and $t_{K+2}$. Computations performed by Gourdon and Demichel give us these values for the specific close pair we are considering (see Table 21). It is then eventually possible to compute a lower bound for $\Lambda$, provided that conditions of Theorem $\mathrm{A}$ are satisfied. Checking that the zeros are simple is not necessary as stated in Section 2. The only remaining problem is to check that computed values for $t_{K-1}, t_{K}, t_{K+1}$ and $t_{K+2}$ are correct, that is to say that no intermediate zero is missing. This point is implicit in computational verifications of the Riemann hypothesis but here an independent verification is necessary, since Gourdon and Demichel's computations are not officially certified.

Modern computational verifications of the Riemann hypothesis have been greatly simplified by Turing's approach to the problem Tur53, although some errors of detail were later corrected by Lehman Leh70. The main interest of this method is that it only involves evaluations of $\zeta$ on the critical line.

First, let us recall some classical definitions. For $t>0$, we define the functions $\theta(t)$ and $Z(t)$ by $\theta(t)=\operatorname{Arg}\left\{\Gamma\left(\frac{1}{4}+\frac{1}{2} i t\right)\right\}-\frac{t}{2} \log \pi$ and $Z(t)=\exp (i \theta(t)) \zeta\left(\frac{1}{2}+i t\right)$.

Then the function $Z(t)$, called the Hardy function, is real and $\zeta(1 / 2+i t)=0$ if and only if $Z(t)=0$. The Riemann-Siegel formula gives a practical way to compute $Z(t)$. The function $\theta(t)$ is computed by its asymptotic expansion for large $t$. For $n \geq 0$, the $n$-th Gram point is defined to be the unique solution greater than 7 of $\theta\left(g_{n}\right)=n \pi$. A Gram point $g_{n}$ is said to be regular if $(-1)^{n} Z\left(g_{n}\right)>0$, and irregular 
otherwise. A Gram block is an interval $\left[g_{n}, g_{n+k}\right)$ with $k \geq 1$ such that $g_{n}$ and $g_{n+k}$ are regular Gram points, while the Gram points $g_{n+k^{\prime}}$ are irregular for all $k^{\prime}$ with $1 \leq k^{\prime}<k$. Corresponding to the points $g_{n}$, we define a companion sequence $h_{n}$ of real numbers such that

- $h_{n}$ can be positive, zero or negative;

- if $g_{n}$ is a regular Gram point, then $h_{n}=0$;

- the property $(-1)^{n} Z\left(g_{n}+h_{n}\right)>0$ holds for all $n$;

- the series $g_{n}+h_{n}$ is monotonically increasing.

Finally, we denote by $N(T)$ as usual the number of solutions to $\zeta(\sigma+i t)=0$ with $0 \leq t<T$, and $0<\sigma<1$. We then have the following.

Theorem C. Let $n$ be a positive integer such that $g_{n}$ is a regular Gram point. If there exist integers $k, k^{\prime}>0$ such that $g_{n-k}>168 \pi$ and

$$
\begin{aligned}
\frac{2.30+0.128 \log \left(\frac{g_{n}}{2 \pi}\right)+\sum_{j=1}^{k-1} h_{n-j}}{g_{n}-g_{n-k}} & <1, \\
\frac{2.30+0.128 \log \left(\frac{g_{n+k^{\prime}}}{2 \pi}\right)+\sum_{j=1}^{k^{\prime}-1} h_{n+j}}{g_{n+k^{\prime}}-g_{n}} & <1,
\end{aligned}
$$

then we have $N\left(g_{n}\right)=n+1$.

Proof. This theorem is proved for the general case of Dirichlet $L$-series in Rum93, Prop. 3].

Thus, Theorem $\mathrm{C}$ can be used to determine exactly the number of solutions to $\zeta(\sigma+i t)=0$ with $0 \leq t<g_{n}$ and $0<\sigma<1$, provided that $g_{n}$ is a regular Gram point and that a companion series $h_{m}$ has been computed for $n-k+1 \leq m \leq$ $n+k^{\prime}-1$. To apply Theorem $\left[\right.$, the values $k$ and $k^{\prime}$ can be arbitrary, i.e., no hypothesis is required on the eventual regularity of either the Gram points $g_{n-k}$ or $g_{n+k^{\prime}}$, or for any intermediate Gram points (except $g_{n}$ ).

It has also to be noted that numerical values 2.30 and 0.128 involved in Theorem C can be improved. Recently, Trudgian [Tru09] claimed that they can be replaced by 1.41 and 0.0054 , respectively. However, this improvement, from a computational point of view, leads to only a minor decrease in complexity. Indeed, when checking the Riemann hypothesis, most of the time is spent evaluating the function $Z$ at various points in order to locate changes of signs. Thus, in such computations, the impact of Trudgian's result is quite limited.

Saouter made an independent verification of the computed values for zeros in an interval of $200 \mathrm{Gram}$ points around the critical pair $\left(t_{K}, t_{K+1}\right)$. Since few zeros had to be checked, the Riemann-Siegel formula was used (see BBC00), unlike in Gourdon's and Demichel's work. To ensure great precision in computed zeros, three correcting terms were also used. Computations were made by the MPFR [FHL+07] multiprecision package. Computed values for zeros in this second verification were in complete agreement with those computed by Gourdon and Demichel. A companion series $h_{n}$ was computed and, setting $k=k^{\prime}=35$ in Theorem Cl computations showed that Turing's condition holds at least for all Gram points in the interval $\left[g_{K-65}, g_{K+65}\right]$. Thus, no zero is missing in Table 2

Reminding ourselves that we have $x_{n}=2 t_{n}$ for all $n \geq 0$, we can now use Theorem B] We obtain $G_{K} \leq 379.1995$ and $\left(x_{K+1}-x_{K}\right)^{2} \times G_{K} \leq 3.47471 \times 10^{-8}$. Thus, $\left(x_{K}, x_{K+1}\right)$ is a Lehmer pair and Theorem $\mathrm{A}$ gives $\lambda_{K}>-1.14541 \times 10^{-11}$. 
TABLE 3. Probability of appearance of Lehmer pairs.

\begin{tabular}{|r|r|r|r|r|}
\hline Lower bound & $\delta$ & $M$ & Probability & Reference \\
\hline$-5.90 \times 10^{-9}$ & $1.09 \times 10^{-4}$ & $1.5 \times 10^{9}$ & 0.049 & [OSV93] \\
\hline$-2.63 \times 10^{-9}$ & $1.45 \times 10^{-4}$ & $5 \times 10^{9}$ & 0.994 & Odl00] \\
\hline$-1.15 \times 10^{-11}$ & $2.084 \times 10^{-5}$ & $1 \times 10^{14}$ & 0.629 & This paper \\
\hline
\end{tabular}

\section{FinAl REMARKS}

The result we obtained was enabled by the discovery of a close pair of zeros for the Riemann $\zeta$ function by Gourdon and Demichel GD04. In the conclusion of [Odl00], Odlyzko expresses the idea that systematic computations of zeros should produce still better and better lower bounds for $\Lambda$. A question then comes to mind: is the current Lehmer pair we used exceptional or not? The exact distribution law of zeros of the Riemann $\zeta$ function is unknown, but it is conjectured to be the same as that of eigenvalues of matrices of the Gaussian unitary ensemble. This conjecture comes from practical observations, but it agrees with Hilbert and Pólya's conjecture that the zeros of the Riemann $\zeta$ function correspond to eigenvalues of some positive linear operator.

The Gaussian unitary ensemble has been widely studied in physics. Odlyzko compared the results from this model to the numerical data of zeros of the Riemann $\zeta$ function Odl87. For instance, the GUE conjecture implies that if you define $\delta_{n}=$ $\left(t_{n+1}-t_{n}\right) \frac{\log \frac{t_{n}}{2 \pi}}{2 \pi}$, then the probability that the minimal value, amongst $M$ consecutive values, for $\delta_{n}$ is less than $\alpha M^{-1 / 3}$, is approximately equal to $1-\exp \left(-\pi^{2} \alpha^{3} / 9\right)$, provided that $\alpha^{3} / M$ is small. From this relation, and taking into account the number of zeros which have been computed, it is possible to give a quantative appreciation of close Lehmer pairs that have been used in this and earlier work. These values are recorded in Table 3. Thus, we can observe that, while in COSV93, the Lehmer pair that has been used was rather exceptional, the one used in Odl00 was almost totally predictable. The probability of the pair used in this paper lies between these two: neither exceptional, nor certain. The strategies to obtain these pairs differ. In COSV93, the pair was obtained by a complete enumeration of the first $1.5 \times 10^{9}$ zeros of the Riemann $\zeta$ function. In Odl00, the pair considered was obtained by computing $5 \times 10^{9}$ zeros around the $10^{20}$-th zero. Finally, the computation in this paper is based on a complete enumeration of the first $10^{14}$ zeros, performed by Gourdon and Demichel GD04. If $10^{14}$ zeros around the $10^{20}$-th zero had been computed, then, with the same probability as in this paper, we could have expected to obtain a close pair with $\delta \simeq 2 \times 10^{-5}$ and thus a lower bound of approximately $-1.1 \times 10^{-12}$ for $\Lambda$. However, it has to be noted that complete enumerations of first zeros of $\zeta$ have widespread applications in computational number theory. For instance, this kind of result can be used to refine knowledge of the prime number distribution [Dus98. This is not the case for isolated computations.

\section{REFERENCES}

[BBC00] J.M. Borwein, D.M. Bradley, and R.E. Crandall. Computational strategies for the Riemann zeta function. J of Comp'l and Applied Math., 121:247-296, 2000. MR1780051 (2001h:11110)

[CNV88] G. Csordas, T.S. Norfolk, and R.S. Varga. A lower bound for the de Bruijn-Newman constant $\Lambda$. Numer. Math., 52:483-497, 1988. MR945095 (89m:30054) 
[COSV93] G. Csordas, A.M. Odlyzko, W. Smith, and R.S. Varga. A new Lehmer pair of zeros and a new lower bound for the de Bruijn-Newman constant $\Lambda$. Electron. Trans. Numer. Anal., 1:104-111, 1993. MR1253639(94k:11098)

[CRV91] G. Csordas, A. Ruttan, and R.S. Varga. The Laguerre inequalities with applications to a problem associated with the Riemann hypothesis. Numer. Algorithms, 1:305-329, 1991. MR 1135299 (93c:30041)

[CSV94] G. Csordas, W. Smith, and R.S. Varga. Lehmer pairs, the de Bruijn-Newman constant $\Lambda$, and the Riemann hypothesis. Constr. Approx., 10:107-129, 1994. MR 1260363 (94k:30061)

[dB50] N.G. de Bruijn. The roots of trigonometric integrals. Duke J. Math., 17:197-226, 1950. MR0037351(12:250a)

[Dus98] P. Dusart. Autour de la fonction qui compte le nombre de nombres premiers. $\mathrm{PhD}$ thesis, Université de Limoges, 1998.

[FHL+07] L. Fousse, G. Hanrot, V. Lefèvre, P. Pélissier, and P. Zimmermann. MPFR: A multipleprecision binary floating-point library with correct rounding. ACM Transactions on Mathematical Software, 33(2):13:1-13:15, June 2007. MR2326955 (2008e:65157)

[GD04] X. Gourdon and P. Demichel. The first $10^{13}$ zeros of the Riemann Zeta function, and zeros computation at very large height. Available at http://numbers.computation.free.fr/Constants/Miscellaneous/zetazeros1e131e24.pdf, 2004.

[KKL09] H. Ki, Y.O. Kim, and J. Lee. On the de Bruijn-Newman constant. Advances in Mathematics, 22:281-306, 2009. MR2531375 (2010d:30033)

[Leh70] R.S. Lehman. On the distribution of zeros of the Riemann zeta-function. Proc. London Math. Soc., 3(20):303-320, 1970. MR0258768(41:3414)

[New76] C.M. Newman. Fourier transforms with only real zeros. Proc. Amer. Math. Soc., 61:245-251, 1976. MR0434982(55:7944)

[NRV92] T.S. Norfolk, A. Ruttan, and R.S. Varga. A lower bound for the de Bruijn-Newman constant $\Lambda$ II. In A.A. Gonchar and E.B. Saff, editors, Progess in Approximation Theory, 403-418. Springer-Verlag, 1992. MR1240792 (94k:30062)

[Odl87] A.M. Odlyzko. On the distribution of spacings between zeros of the zeta function. Math. Comp., 48(177):273-308, 1987. MR866115 (88d:11082)

[Od100] A.M. Odlyzko. An improved bound for the de Bruijn-Newman constant. Numer. Algorithms, 25:293-303, 2000. MR1827160 (2002a:30046)

[OS88] A.M. Odlyzko and A. Schönage. Fast algorithms for multiple evaluations of the Riemann zeta function. Trans. Amer. Math. Soc., 309:797-809, 1988. MR961614 $(89 \mathrm{j}: 11083)$

[Rum93] R. Rumely. Numerical computations concerning the ERH. Math. Comp., 61(203):415440, 1993. MR.1195435 (94b:11085)

[Tit86] E.C. Titchmarsh. The theory of Riemann zeta-function. Oxford Univ. Press, 2nd edition, 1986. MR882550 (88c:11049)

[tR91] H.J.J. te Riele. A new lower bound for the de Bruijn-Newman constant. Numer. Math., 58:661-667, 1991. MR1083527 (92c:30030)

[Tru09] T. Trudgian. Improvements to Turing's method. http://arxiv.org/abs/0903.1885v1, March 2009.

[Tur53] A.M. Turing. Some calculations of the Riemann zeta-Function. Proc. London Math. Soc., 3(3):99-117, 1953. MR0055785 (14:1126e)

[vdL01] J. van de Lune. Unpublished, 2001.

[Wed05] S. Wedeniwski. Zetagrid home page. http://www.zetagrid.net/, 2005.

Institut Telecom Brest, Bretagne, France

E-mail address: Yannick. Saouter@telecom-bretagne.eu

Dassault Systemes, Velizy-Villacoublay, France

E-mail address: xgourdon@gmail.com

Hewlett-Packard France, Les Ulis, France

E-mail address: patrick.demichel@hp.com 